OPEN

SUBJECT AREAS:

TWO-DIMENSIONAL

MATERIALS

SYNTHESIS OF GRAPHENE

Received

12 October 2014

Accepted

28 November 2014

Published

19 December 2014

Correspondence and requests for materials should be addressed to

G.K. (kalita.golap@ nitech.ac.jp)

\section{Visualizing copper assisted graphene growth in nanoscale}

\author{
Mohamad Saufi Rosmi' , Mohd Zamri Yusop ${ }^{1,3}$, Golap Kalita ${ }^{1,2}$, Yazid Yaakob' ', Chisato Takahashi' \\ \& Masaki Tanemura'
}

\begin{abstract}
${ }^{1}$ Department of Frontier Materials, Nagoya Institute of Technology, Gokiso-cho, Showa-ku, Nagoya 466-8555, Japan, ${ }^{2}$ Center for Fostering Young and Innovative Researchers, Nagoya Institute of Technology, Gokiso-cho, Showa-ku, Nagoya, 466-8555, Japan, ${ }^{3}$ Department of Materials, Faculty of Mechanical Engineering, Universiti Teknologi Malaysia, 81310 Skudai, Johor, Malaysia.
\end{abstract}

Control synthesis of high quality large-area graphene on transition metals (TMs) by chemical vapor deposition (CVD) is the most fascinating approach for practical device applications. Interaction of carbon atoms and TMs is quite critical to obtain graphene with precise layer number, crystal size and structure. Here, we reveal a solid phase reaction process to achieve $\mathrm{Cu}$ assisted graphene growth in nanoscale by in-situ transmission electron microscope (TEM). Significant structural transformation of amorphous carbon nanofiber $(\mathrm{CNF})$ coated with $\mathrm{Cu}$ is observed with an applied potential in a two probe system. The coated $\mathrm{Cu}$ particle recrystallize and agglomerate toward the cathode with applied potential due to joule heating and large thermal gradient. Consequently, the amorphous carbon start crystallizing and forming $\mathrm{sp}^{2}$ hybridized carbon to form graphene sheet from the tip of $\mathrm{Cu}$ surface. We observed structural deformation and breaking of the graphene nanoribbon with a higher applied potential, attributing to saturated current flow and induced Joule heating. The observed graphene formation in nanoscale by the in-situ TEM process can be significant to understand carbon atoms and $\mathrm{Cu}$ interaction.
S ince the first isolation of graphene, two dimensional materials has been gaining significant attention in various prospective of device applications ${ }^{1-6}$. Over the last few years, significant development on graphene synthesis technology has been witnessed, bringing new ideas of innovation for device integration ${ }^{7-12}$. Synthesis of large-area high quality graphene by atmospheric or low pressure CVD process is one of the most fascinating aspect in graphene technology ${ }^{10-17}$. Continuous single layer graphene film and single crystal domains have been synthesized by the CVD process on TMs substrate ${ }^{10-12,15-21}$. In prospect to achieve control graphene growth, understanding of carbon atoms and metal surface interaction during growth process is of great interest. Theoretical and experimental studies were progressing to understand graphene formation process on TMs surface by a CVD technique ${ }^{22-25}$. Exploring carbon atoms interaction with TMs in nanoscale can be also significant to visualize graphene growth process. Subsequently, it can give an insight in a solid source reaction process of graphene growth in presence of TMs.

In-situ TEM study of carbon materials is of great interest to explore the physical properties and chemical structural transformation process in nanoscale ${ }^{26-30}$. Structural transformation of carbon nanotubes (CNTs) has been considerably investigated by the in-situ $\mathrm{TEM}^{31-34}$. Previously, Ichihashi et al. has reported transformation of Fe-doped amorphous carbon into graphitic tubular structure after annealing at $650-900^{\circ} \mathrm{C}^{32}$. Again, electrochemical sodiation process and microstructure evolution of individual Co9S8-filled CNTs have been explored by in-situ TEM ${ }^{33}$. Similarly, we have observed polycrystalline carbon nanofiber (CNF) transformation to an onionring-like graphitized structure by an in-situ TEM field emission (FE) process ${ }^{34}$. Recently, we observed FE-induced structural change of $\mathrm{Fe}$ incorporated CNF by in-situ $\mathrm{TEM}^{35}$. In the FE measurements, dramatic transformation of the amorphous Fe-incorporated $\mathrm{CNF}$ was observed to form a hollow graphitic nanostructure with migration of $\mathrm{Fe}$ particle. The significant structural change was obtained owing to bulk diffusion process of carbon atoms in highly soluble Fe nanoparticles with joule heating effect. It is quite interesting to investigate how the solid phase reaction will be occur in case of low carbon solubility TMs. In this prospect, we demonstrated structural transformation mechanism of Au-incorporated CNF to determine catalytic ability of Au for graphitization ${ }^{36}$.

However, any of the previous in-situ TEM studies did not realize graphene sheet formation in presence of TMs. Considering the possibility of graphene growth by in-situ TEM, we explored the electrical conduction behavior of $\mathrm{CNF}$ coated $\mathrm{Cu}$ in a two probe system. The coated $\mathrm{Cu}$ particle recrystallize and agglomerate with applied electric field and move toward cathode. Consequently, the amorphous carbon atoms of CNF convert to sp ${ }^{2}$ hybridized 

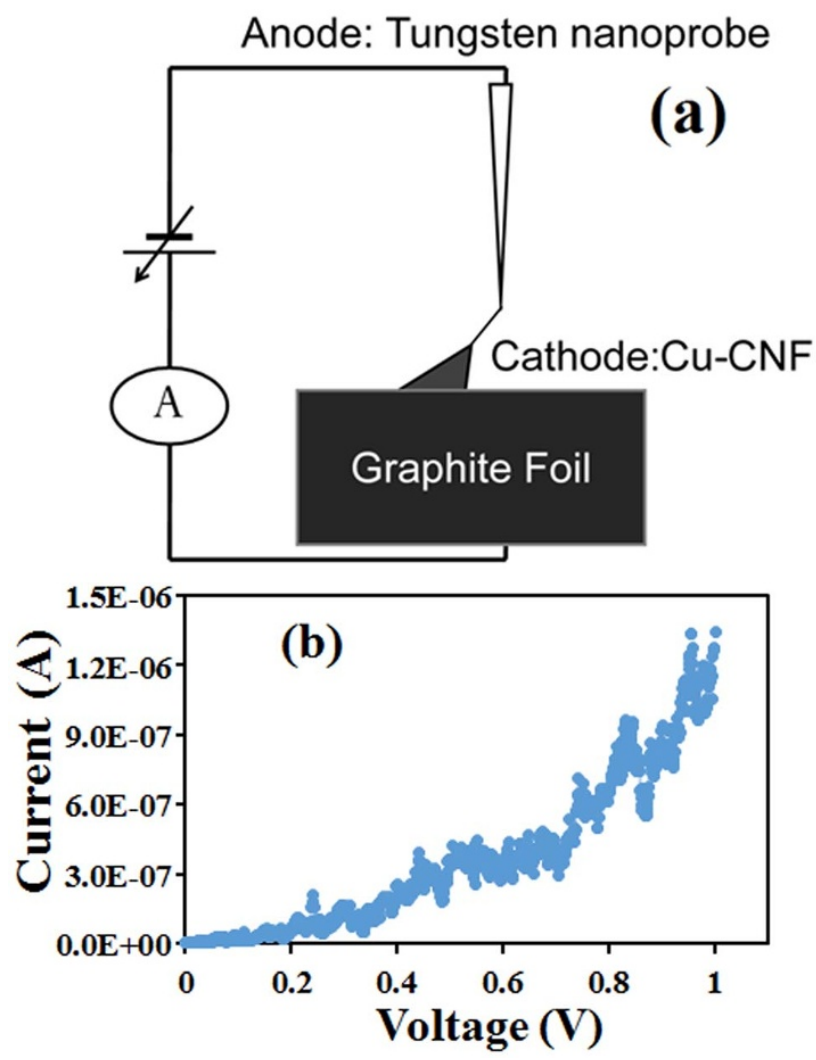

Figure $1 \mid$ (a) Schematic diagram of the in-situ TEM I-V measurement setup. (b) I-V characteristic of the Cu coated CNF as measured by the two probe system at lower applied potential. carbon in presence of $\mathrm{Cu}$ to form graphene structure. In what follows, we demonstrate $\mathrm{Cu}$ and carbon atoms interaction in nanoscale, achieving graphene nanoribbon growth by in-situ TEM.

\section{Results and discussion}

Fig. 1a shows a schematic diagram of the in-situ current-voltage (IV) measurement setup. A tungsten nanoprobe controlled by nanomanipulator was used as anode in the in-situ experimental process. On the other hand, CNF prepared on a graphite foil by argon ions $(\mathrm{Ar}+)$ irradiation at room temperature as reported elsewhere was taken as the cathode ${ }^{34}$. The CNF prepared by ion irradiation was coated with $\mathrm{Cu}$ to investigate the $\mathrm{Cu}$ and carbon interaction with applied bias potential. The structural transformation of the $\mathrm{Cu}$ coated $\mathrm{CNF}(\mathrm{Cu}-\mathrm{CNF})$ of the cathode was visualized by in-situ TEM in nanoscale by applying bias potential. The pressure in the specimen chamber was about $10^{-5} \mathrm{~Pa}$ in the I-V measurement process. We investigated the morphological change with lower and higher bias voltage. Fig. $1 \mathrm{~b}$ shows an I-V characteristic of the $\mathrm{Cu}-$ $\mathrm{CNF}$ as measured by the two probe system. The high resistance of amorphous CNF significantly affect the flow of current (maximum current flow $\sim 10^{-5} \mathrm{~A}$ ) at lower applied potential.

Fig. 2a shows a TEM image of the Cu-CNF sample created on the graphitic plate. The image shows that CNF are around $100 \sim 500 \mathrm{~nm}$ in length, fully coated with polycrystalline $\mathrm{Cu}$ particles. Fig. $2 \mathrm{~b}$ shows a TEM image at the contact of nanoprobe and $\mathrm{Cu}-\mathrm{CNF}$. In a low current flow through the $\mathrm{Cu}-\mathrm{CNF}$ also induced joule heating effect was observed. Poor crystalline graphene sheet was formed from the surface contact with resistive joule heating effect. Fig. $2 c$ shows a TEM image of low crystalline few-layers graphene sheet. The amorphous carbon from the CNF recrystallize to form $\mathrm{sp}^{2}$ carbon in presence of $\mathrm{Cu}$, while the $\mathrm{Cu}$ particles start agglomerating toward the cathode. The interesting effect of graphene formation in presence of

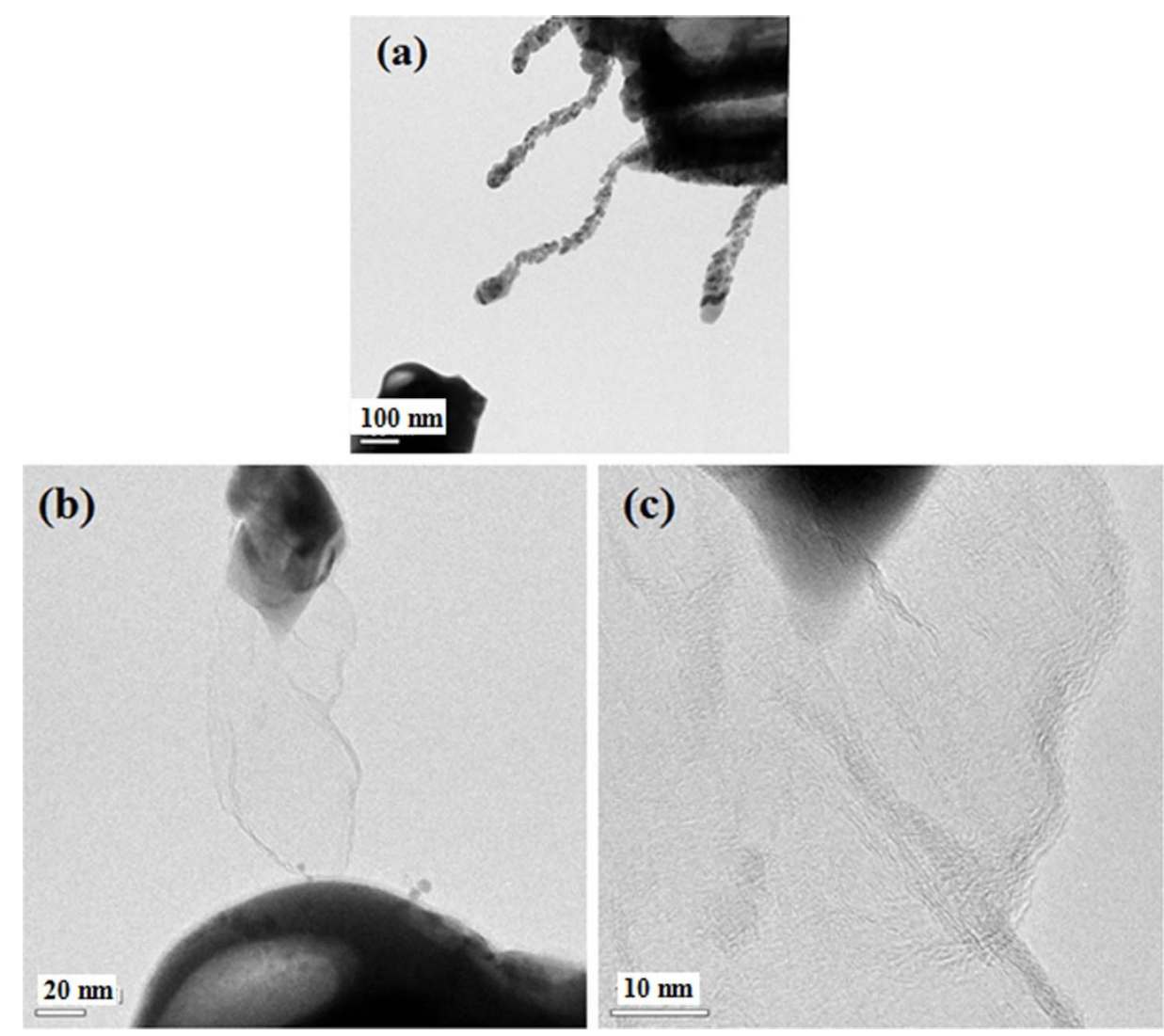

Figure 2 $\mid$ TEM image of (a) Cu-CNF sample created on the graphitic plate, (b) graphitization at the contact of nanoprobe and Cu-CNF after applying low bias potential. (c) Higher resolution image of the low crystalline few-layers graphene sheet. 

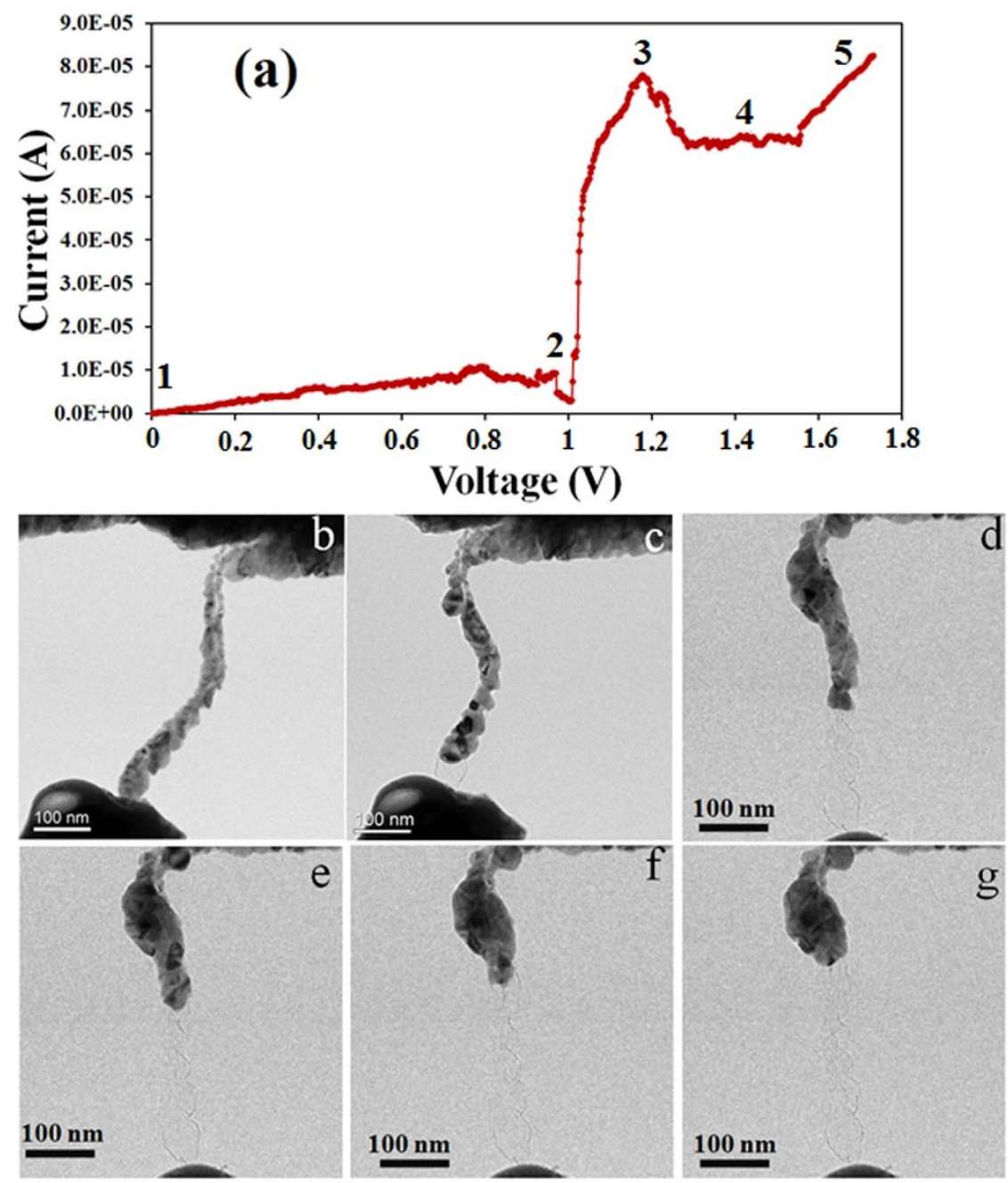

Figure $3 \mid$ (a) I-V curve of the Cu-CNF with higher bias voltage in the two probe system $(0-1.8 \mathrm{~V})$. (b) - (g) TEM images of the Cu-CNF with change in applied potential from $0.0-1.8 \mathrm{~V}$ as that of the I-V characteristics. TEM images presenting the structural transformation with gradual increase in applied potential. Formation of a graphene nanoribbon across the cathode and anode is obtained with a length of around $\sim 300 \mathrm{~nm}$ and width $\sim 50 \mathrm{~nm}$.

$\mathrm{Cu}$ was further investigated with higher applied potential to understand the growth process.

Fig. 3a shows an I-V curve of the Cu-CNF with higher bias voltage in the two probe system. It was observed that there was no significant current flow through the $\mathrm{CNF}$ at an applied voltage up to $1 \mathrm{~V}$. There is a sharp rise in current flow with significant drop of resistance from $340 \mathrm{~K} \Omega$ to $15.3 \mathrm{~K} \Omega$. Applying more than $1 \mathrm{~V}$ saturated current flow was obtained. We observed significant structural transformation of the $\mathrm{Cu}-\mathrm{CNF}$ with higher current flow and induced joule heating. Fig. 3b-g shows TEM image of the $\mathrm{Cu}-\mathrm{CNF}$ with change in applied potential from $0.0-1.8 \mathrm{~V}$ as that of the $\mathrm{I}-\mathrm{V}$ characteristics. As shown in fig. 3b, initially the $\mathrm{Cu}-\mathrm{CNF}$ is connected to tungsten nanoprobe to investigate the structural transformation with gradual increase in applied potential. Fig. $3 \mathrm{c}$ shows that with increase in bias voltage graphene like structure were formed with $\mathrm{Cu}$ particles moving toward cathode from nanoprobe contact. Increase the applied voltage much further, nanoribbon like graphene structure grow with agglomeration of $\mathrm{Cu}$ toward cathode as shown in fig. $3 \mathrm{c}-\mathrm{g}$. Finally, we achieve to grow a graphene nanoribbon of the length around $\sim 300 \mathrm{~nm}$ and width $\sim 50 \mathrm{~nm}$ by the in-situ TEM. The observed growth process of graphene nanoribbon like structure with induced joule heating effect is significant to understand $\mathrm{Cu}$ interaction with carbon atoms. The observed graphene growth phenomena is considerably different than that of carbon nanotube growth in presence of Fe nanoparticle by an in-situ TEM field emission process. In our previous study of Fe-incorporated CNF case, Joule heating caused by the FE-process and CNT-like hollow structure was obtained with electromigration of Fe particles ${ }^{35}$. We have also performed additional two probe contact I-V measurement for Fe-incorporated CNF similar to that of $\mathrm{Cu}-\mathrm{CNF}$. In this experiment also we observed formation of bamboo-like CNTs structure with migration of Fe particles (supplementary information figure S2). The growth of graphene nanoribbon in case of $\mathrm{Cu}-\mathrm{CNF}$ is explained as follows. In the two probe system current flows across the anode and cathode, where a thermal gradient can generate at the contact of anode and CNF tips. The thermal gradient across the cathode and anode act as the driving force for migration of $\mathrm{Cu}^{37}$. Subsequently, $\mathrm{Cu}$-induced graphitization and graphene growth occurs from the amorphous CNF due to joule heating. Carbon atoms can absorb or diffuse on $\mathrm{Cu}$ surface rather than bulk diffusion in the graphene formation process. The ability to form different allotropes of $\mathrm{sp}^{2}$ crystalline carbon significantly depends on the carbon solubility with various transition metals and affinity. Considering the fact that Fe has much higher carbon affinity than that of $\mathrm{Cu}$, the reaction and graphitization process can be significantly different. The filled $3 \mathrm{~d}$-electron shell $[\mathrm{Ar}] 4 \mathrm{~s}^{1} 3 \mathrm{~d}^{10}$ of $\mathrm{Cu}$ suggests low reactivity with carbon and not assisting formation of carbide phase $^{38}$. Thus, the reaction of carbon atoms and transition metals is a crucial factor in nanocarbon formation. In this prospect, carbon and $\mathrm{Cu}$ interaction in graphene growth was further investigated at the edge of nanoparticle by high resolution TEM studies. 


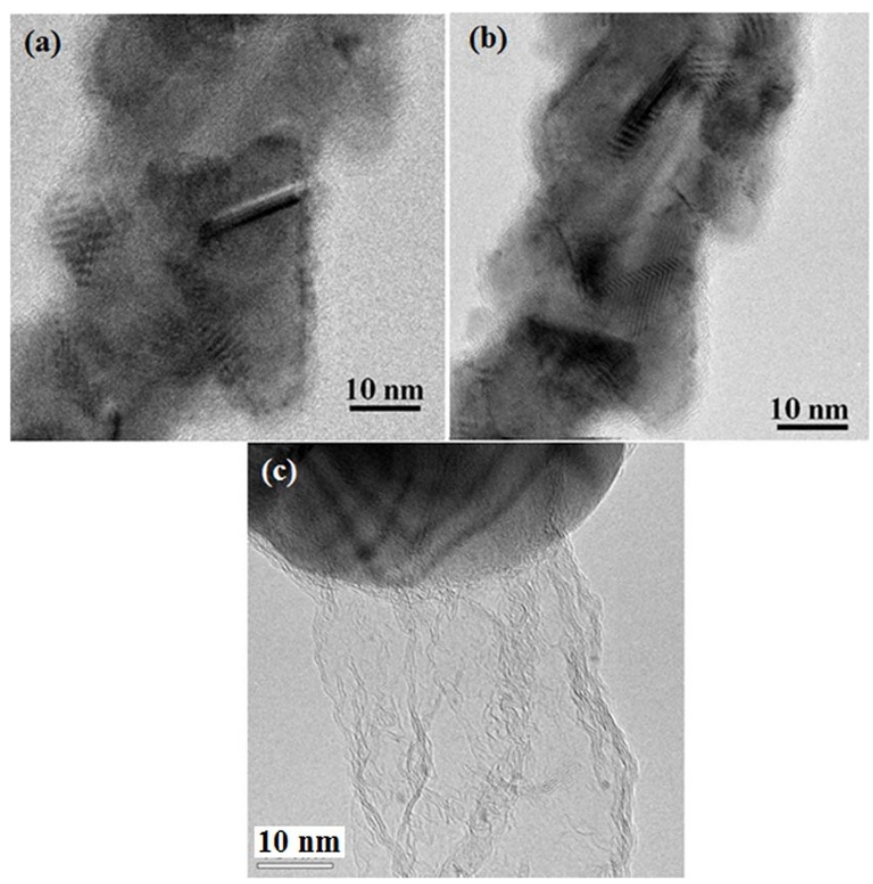

Figure 4| TEM image of the Cu structure (a) before and (b) after applied bias voltage. (c) TEM image at the tip of the $\mathrm{Cu}$ grain, presenting the dissolution of carbon atoms to from graphene nanoribbon structure.

Fig. $4 \mathrm{a}-\mathrm{b}$ shows TEM image of the $\mathrm{Cu}$ structure before and after applied bias voltage. The thermally evaporated $\mathrm{Cu}$ at room temperature shows polycrystalline structure with low crystallinity. Recrystallization of $\mathrm{Cu}$ occurs with induced joule heating during the applied bias voltage. The transformation of polycrystalline $\mathrm{Cu}$ structure and crystalline nature is shown in fig. $4 \mathrm{~b}$. The crystalline plane of $\mathrm{Cu}$ grain became more pronounced with recrystallization due to heating effect. While, we observed poorly graphitized layer structures at the surrounding of the polycrystalline $\mathrm{Cu}$ grains. This result suggest that with joule heating effect the carbon atoms can absorb on $\mathrm{Cu}$ grains to recrystallize and form $\mathrm{sp}^{2}$ hybridized carbon. Increasing the bias voltage and thereby the joule heat, absorb carbon atoms significantly crystalize to create graphene structure. Fig. $4 \mathrm{c}$ shows a TEM image at the tip of $\mathrm{Cu}$ grain. Remarkably, the polycrystalline $\mathrm{Cu}$ grains starts agglomerating toward the $\mathrm{Cu}$ rich cathode with joule heating. In the meantime, the absorbed carbon atoms desorbed from the $\mathrm{Cu}$ surface to create highly crystalline graphene structure. We include a video visual of the graphene growth process observed by the in-situ TEM study (Multimedia view 1).

Fig. 5a shows a TEM image after complete growth of the graphene nanoribbon. The result can be significant to understand $\mathrm{Cu}$ and carbon atoms interaction in nanoscale for graphene growth process. The obtained graphene structure was further confirmed by high resolution TEM images. Fig. 5b-c shows TEM studies of sheet like structure of the synthesized graphene ribbon, in contrast to hollow tube like structure of previous reports. The carbon atoms created $\mathrm{sp}^{2}$ hybridized structure catalyzed by $\mathrm{Cu}$ to form a sheet like structure rather than CNT. An inter-planer spacing of about $0.34 \mathrm{~nm}$ is estimated for the graphene layers, corresponding to graphite (0002) spacing. In most of the previous studies, structural transformation of CNF and CNTs by in-situ TEM has been demonstrated. However, $\mathrm{Cu}$ assisted graphene growth by in-situ TEM was not achieved. The interesting results observed in these studies can provide more light on $\mathrm{Cu}$ and carbon interaction for graphene growth process.

Further, effect of higher applied potential across the cathode and anode after complete growth of the graphene nanoribbon was investigated by the in-situ TEM. The nature of graphene-metal contact has significant importance to investigate the Joule heating effect, current

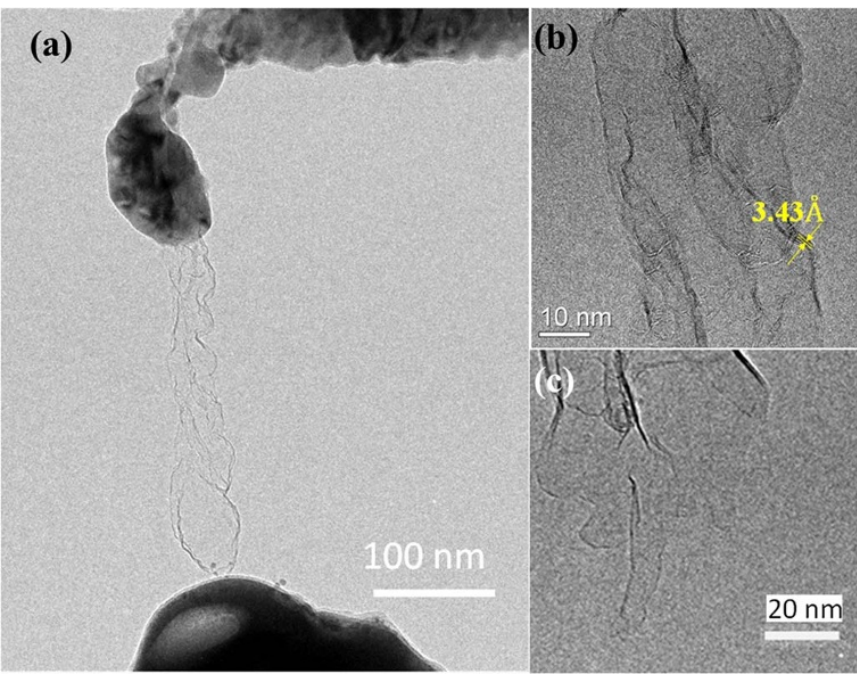

Figure 5 | TEM image (a) after complete growth of the graphene nanoribbon, (b), (c) sheet like structure of the synthesized graphene ribbon, in contrast to hollow tube like structure. The carbon atoms created $\mathrm{sp}^{2}$ hybridized structure catalyzed by $\mathrm{Cu}$ to form a sheet like structure rather than $\mathrm{CNT}$.

crowding and thermoelectric heating process in electronic devices. According to general prospective Joule heating can be explained from a simple semi-classical picture in which electrons collide with the atoms of a conductor, generating heat locally at low dimension. It has been also observed that stable heating up to $2000 \mathrm{~K}$ occurred in an individual multiwalled carbon nanotube induced by field emission current ${ }^{39}$. However, in this case, we did not observe evaporation of $\mathrm{Cu}$ by joule heating effect at a higher vacuum, which signifies less induced joule heating. Fig. 6 a shows I-V characteristic with a higher applied potential across the anode and cathode. We observe gradual increase in current flow and structural transformation amorphous carbon catalyzed by $\mathrm{Cu}$ to form graphene structure. However, the graphene nanoribbon broke at middle part after growth of around $\sim 300 \mathrm{~nm}$ in length as shown in fig. 6b-c. A saturation current of $0.119 \mathrm{~mA}$ was flown through the graphene nanoribbon with an applied bias potential of $2 \mathrm{~V}$. A sharp decrease in current was observed with the saturation current flow and increase in applied voltage. Fig. $6 \mathrm{c}$ shows the TEM image of the broken and disconnected graphene sheets. We also include a video clip for the graphene deformation process as observed by the in-situ TEM study (Multimedia view 2). The breaking of the graphene can be explained with generation of high joule heat with flow of saturated current at low dimension. This indicate that significant amount heat can generate at the low dimensional metal-metal contact and thereby affecting the highly stable and crystalline graphene based materials.

\section{Conclusion}

In conclusion, we have revealed the solid phase reaction process of $\mathrm{Cu}$ and carbon atoms to grow graphene nanoribbon by in-situ TEM. Structural transformation of amorphous CNF coated with $\mathrm{Cu}$ was investigated in nanoscale with applied bias in a two probe system. Significant interaction of $\mathrm{Cu}$ and carbon atoms was visualized to achieve $\mathrm{Cu}$-assisted graphene structure formation. The coated $\mathrm{Cu}$ particles recrystallize with applied potential and agglomerate toward the cathode with joule heating effect and thermomigration. In this process, the amorphous carbon of CNF also started crystallizing and forming $\mathrm{sp}^{2}$ hybridized carbon to form graphene nanoribbon activated by the $\mathrm{Cu}$ particles. We were able to grow the graphene nanoribbon around $\sim 300 \mathrm{~nm}$ in length across the cathode and anode of in-situ TEM. The observed graphene formation in nanoscale by the in-situ TEM process can be significant to understand carbon atoms 

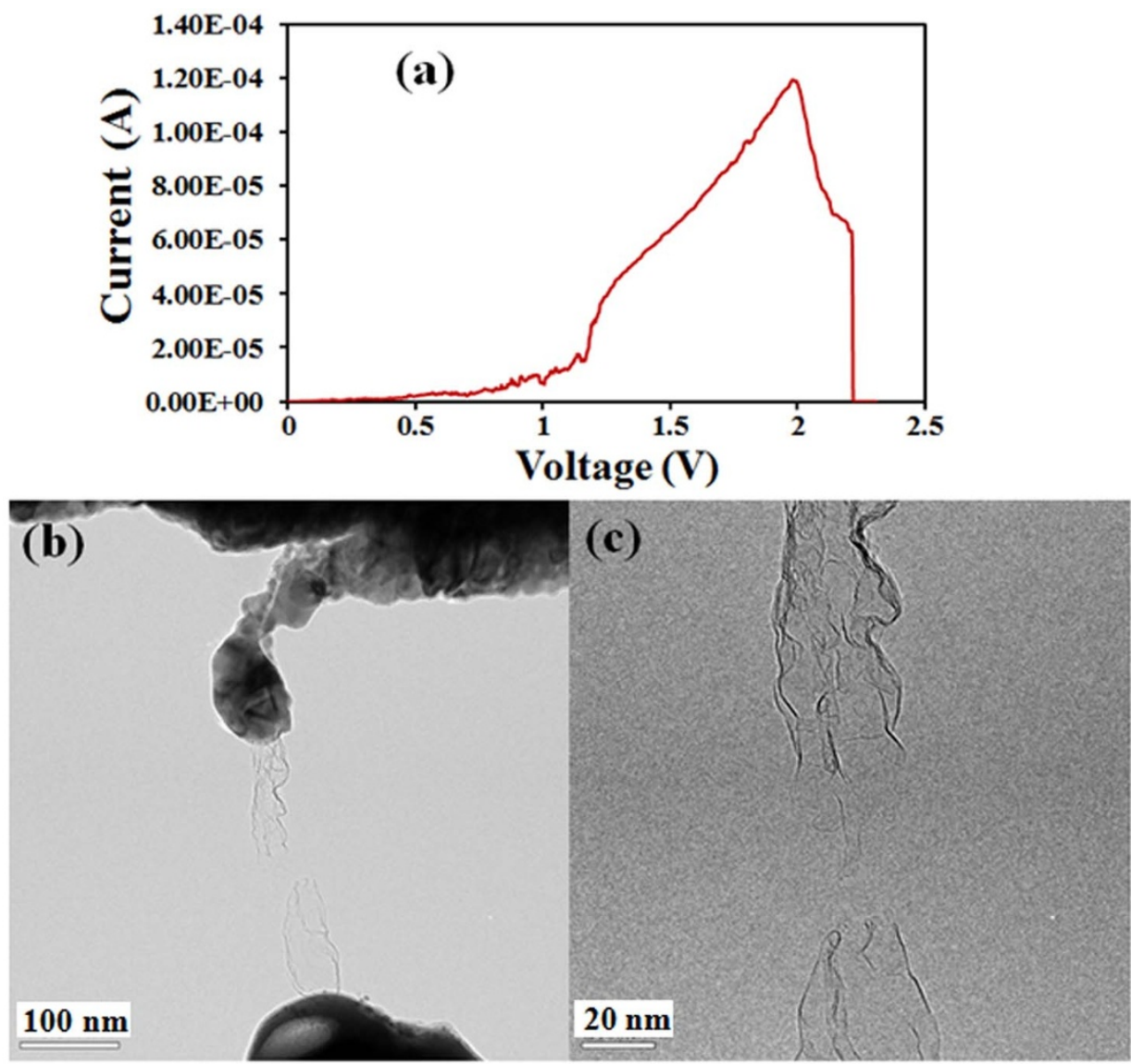

Figure 6 (a) I-V characteristic with a higher applied potential across the anode and cathode. (b) TEM image of breaking the graphene at middle part after growth of around $\sim 300 \mathrm{~nm}$ in length. (c) Higher resolution TEM image of the broken and disconnected graphene sheets.

and $\mathrm{Cu}$ interaction. We also observed deformation and broking of the graphene nanoribbon with high applied potential attributing to current crowding and Joule heating effect in nanoscale.

\section{Methods}

Synthesis of CNF by ion irradiation. For the CNF preparation, we used a commercially available graphite foils with a dimension of $5 \mathrm{~mm} \times 20 \mathrm{~mm} \times 100 \mu \mathrm{m}$ and Kaufmann-type ion gun (Iontech. Inc. Ltd., model 3-1500-100FC) for growing $\mathrm{CNF}$. The oblique $\mathrm{Ar}^{+}$bombardment is known to be more suitable for the growth of ion-induced CNF than the normal incidence. The edges of the graphite foils were irradiated with argon ions $\left(\mathrm{Ar}^{+}\right)$at 45 degree from normal to the surface for $45 \mathrm{~min}$ at room temperature. The diameter and ion beam energy employed for this experiment were $6 \mathrm{~cm}$ and $1 \mathrm{keV}$, respectively. The basal and working pressures were $1.5 \times 10^{-5}$ and $2.0 \times 10^{-2} \mathrm{~Pa}$, respectively. The growth mechanism of ion-induced CNF was explained elsewhere in detail ${ }^{34}$.

$\mathrm{Cu}$ coated CNF sample preparation. $\mathrm{Cu}$ was coated by thermal evaporation on the fabricated CNF on graphite foil. Cu coating was carried out with ULVAC VPC-260F thermal evaporator. Later the $\mathrm{Cu}$ coated graphite plate was cut into small sizes of $2 \mathrm{~mm}$ width and directly mounted on the TEM sample holder without any additional post-treatment to observe the structure transformation and crystallinity of $\mathrm{Cu}-\mathrm{CNF}$.

In-situ TEM and I-V measurements. The TEM analysis was performed by JEOL JEM-2010 with vacuum chamber pressure less than $2.5 \times 10^{-5} \mathrm{~Pa}$. We used a TEM sample holder (JEOL; EM-Z02154T) with a tungsten nanoprobe controlled by nanomanipulator and electrical biasing equipment which allowing us to observe the morphological transformation of the Cu coated CNF sample. The crystalline structure of $\mathrm{Cu}$ and amorphous carbon with applied potential of the prepared $\mathrm{Cu}$ CNF is explored.

1. Novoselov, K. S. et al. Electric field effect in atomically thin carbon films. Science 306, 666 (2004).

2. Geim, A. K. \& Novoselov, K. S. The rise of graphene. Nat. Mater. 6, 183-191 (2007).

3. Morozov, S. V. et al. Giant intrinsic carrier mobilities in graphene and its bilayer. Phys. Rev. Lett. 100, 016602 (2008).
4. Nair, R. R. et al. Fine structure constant defines visual transparency of graphene. Science 320, 1308-1308 (2008).

5. Bolotin, K. I., Ghahari, F., Shulman, M. D., Stormer, H. L. \& Kim, P. Observation of the fractional quantum Hall effect in graphene. Nature 462, 196-199 (2009).

6. Lin, Y. et al. Wafer-scale graphene integrated circuit. Science 332, 1294-1297 (2011).

7. Novoselov, K. S. et al. Two-dimensional atomic crystals. Proc. Natl. Acad. Sci. 102, 10451 (2005)

8. Berger, C. et al. Electronic confinement and coherence in patterned epitaxial graphene. Science 12, 1191-1196 (2006).

9. Stankovich, S. et al. Graphene-based composite materials. Nature 442, 282-286 (2006).

10. Bae, S. et al. Roll-to-roll production of 30-inch graphene films for transparent electrodes. Nat. Nanotechnol. 5, 574-578 (2010).

11. Kim, K. S. et al. Large-scale pattern growth of graphene films for stretchable transparent electrodes. Nature 457, 706-710 (2009).

12. Li, X. S. et al. Large-area synthesis of high-quality and uniform graphene films on copper foils. Science 324, 1312-1314 (2009).

13. Kalita, G., Masahiro, M., Uchida, H., Wakita, K. \& Umeno, M. Graphene constructed carbon thin films as transparent electrodes for solar cell applications. J. Mater. Chem. 20, 9713- (2010).

14. Yusop, M. Z., Kalita, G., Yaakob, Y., Takahashi, C. \& Tanemura, M. Field emission properties of chemical vapor deposited individual graphene. Appl. Phys. Letts. 104, 093501 (2014).

15. Li, X. et al. Large-area graphene single crystals grown by low-pressure chemical vapor deposition of methane on copper. J. Am. Chem. Soc. 133, 2816-2819 (2011).

16. Sharma, S. et al. Synthesis of graphene crystals from solid waste plastic by chemical vapor deposition. Carbon 72, 66-73 (2014).

17. Vlassiouk, I. et al. Large scale atmospheric pressure chemical vapor deposition of graphene. Carbon 54, 58-67 (2013).

18. Gao, L. et al. Repeated growth and bubbling transfer of graphene with millimetresize single-crystal grains using platinum. Nat. Commun. 3, 699 (2012).

19. Wu, T. et al. Triggering the continuous growth of graphene toward millimetersized grains. Adv. Funct. Mater. 23, 198-203 (2013).

20. Yan, Z. et al. Toward the synthesis of wafer-scale single-crystal graphene on copper foils. ACS Nano 6, 9110-9117 (2012). 
21. Wu, W. et al. Growth of single crystal graphene arrays by locally controlling nucleation on polycrystalline $\mathrm{Cu}$ using chemical vapor deposition. Adv. Mater. 23, 4898-4903 (2011).

22. Prezzi, D. Edge structures for nanoscale graphene islands on $\mathrm{Co}(0001)$ surfaces. ACS Nano 8, 5765-5773 (2014).

23. Puretzky, A. A., Geohegan, D. B., Fan, X. \& Pennycook, S. J. In situ imaging and spectroscopy of single-wall carbon nanotube synthesis by laser vaporization. Appl. Phys. Letts. 76, 182 (2000).

24. Merino, P. et al. Sublattice localized electronic states in atomically resolved graphene-Pt(111) edge-boundaries. ACS Nano 8, 3590-3596 (2014).

25. Zhang, W., Wu, P., Li, Z. \& Yang, J. First-principles thermodynamics of graphene growth on Cu Surfaces. J. Phys. Chem. C 115, 17782-17787 (2011).

26. Wanga, Z. L., Poncharalb, P. \& Heer de, W. A. Measuring physical and mechanica properties of individual carbon nanotubes by in situ TEM. Jour. Phys. Chem. Sol. 61, 1025 (2000).

27. Xu, Z., Bai, X. D., Wang, E. G. \& Wang, Z. L. Field emission of individual carbon nanotube with in situ tip image and real work function. Appl. Phys. Letts. 87, 163106 (2005)

28. Rao, R., Liptak, D., Cherukuri, T. \& Yakobson, B. I. B. Maruyama, In situ evidence for chirality-dependent growth rates of individual carbon nanotubes. Nat. Mater. 11, 213 (2012).

29. Kaiser, M., Doytcheva, M., Verheijen, M. \& Jonge de, N. In situ transmission electron microscopy observations of individually selected freestanding carbon nanotubes during field emission. Ultramicroscopy 106, 902-908 (2006).

30. Zhang, D. et al. In situ supported $\mathrm{MnOx}-\mathrm{CeOx}$ on carbon nanotubes for the lowtemperature selective catalytic reduction of $\mathrm{NO}$ with $\mathrm{NH}_{3}$. Nanoscale $\mathbf{5}$, 1127-1136 (2013).

31. Karita, M., Asaka, K., Nakahara, H. \& Saito, Y. In situ TEM study on changes in structure and electrical conductance of carbon nanotube-gold contact induced by local joule heating. J. Mater. Sci. 48, 936-940 (2013).

32. Ichihashi, T., Fuiita, J., Ishida, M. \& Ochiai, Y. In situ observation of carbonnanopillar tubulization caused by liquid like iron particles. Phys. Rev. Lett. 92, 215702 (2004).

33. Su, Q. et al. In situ transmission electron microscopy observation of electrochemical sodiation of individual Co9S8-filled carbon nanotubes. ACS Nano 8, 3620-3627 (2014).

34. Zamri, M., Ghosh, P., Hayashi, A., Hayashi, Y. \& Tanemura, M. Structural change of ion-induced carbon nanofibers by electron current flow. J. Vac. Sci. Technol. B 28, C2C9 (2010).

35. Zamri, M. et al. In Situ TEM Observation of Fe-Included Carbon Nanofiber: Evolution of Structural and Electrical Properties in Field Emission Process. ACS Nano 6, 9567-9573 (2012).
36. Takahashi, C., Yaakob, Y., Yusop, M. Z. M., Kalita, G. \& Tanemura, M. Direct observation of structural change in Au-incorporated carbon nanofibers during field emission process. Carbon 75, 277-280 (2014).

37. Barreiro, A. et al. Subnanometer motion of cargoes driven by thermal gradient along carbon nanotubes. Science 320, 775-778 (2008).

38. Mattevi, C., Kim, H. \& Chhowalla, M. A review of chemical vapour deposition of graphene on copper. J. Mater. Chem. 21, 3324-3334 (2011).

39. Purcell, S., Vincent, P., Journet, C. \& Binh, V. T. Hot nanotubes: stable heating of individual Multiwall carbon nanotubes to $2000 \mathrm{~K}$ induced by the field-emission current. Phys. Rev. Lett. 88, 105502 (2002).

\section{Acknowledgments}

The work is supported by the funds for the development of human resources in science and technology, Japan.

\section{Author contributions}

The experiments were carried out by M. R., M. Y., Y.Y. and C.T. discussing with M.T. and G.K. The manuscript was prepared by G.K. and M.T. discussing the results and experimental conditions with all other authors. All authors have given approval to the final version of the manuscript.

\section{Additional information}

Supplementary information accompanies this paper at http://www.nature.com/ scientificreports

Competing financial interests: The authors declare no competing financial interests.

How to cite this article: Rosmi, M.S. et al. Visualizing copper assisted graphene growth in nanoscale. Sci. Rep. 4, 7563; DOI:10.1038/srep07563 (2014).

This work is licensed under a Creative Commons Attribution-NonCommercialNoDerivs 4.0 International License. The images or other third party material in this article are included in the article's Creative Commons license, unless indicated otherwise in the credit line; if the material is not included under the Creative Commons license, users will need to obtain permission from the license holder in order to reproduce the material. To view a copy of this license, visit http:// creativecommons.org/licenses/by-nc-nd/4.0/ 